\title{
Mesenchymal stem cells derived from rat bone marrow (rbm msc): techniques for isolation, expansion and differentiation
}

\begin{abstract}
Mesenchymal stem cells derived from bone marrow have significant capacity for attachment, expansion and multiline age differentiation. This property has been widely used in the clinical practice for treatment of variety of diseases. Stem cell collection and culturing techniques varies for different species. Time taken for the primary cell culture to attain confluency depends on the species from which bone marrow is isolated. This paper describes an easy technique for the isolation and differentiation of mesenchymal stem cells derived from rat bone marrow (r-MSCs) on the basis of their density gradients and plastic adherence property along with the multiline age differentiation potential. After isolation, characterization was performed morphologically by examination under phase contrast microscope and viability detected by cell viability assay. Spindle shaped morphology was exhibited by the attached nucleated cells. Their multiline age differentiation to osteoblast, adiposities, osteoblast and chondroblasts was examined with respective induction medium and confirmed by oil red O, alizarin red and Alcian blue stains respectively. The r-MSCs were cultured and passaged for their expansion and were maintained in suitable environment for long time and can be used for cell based regenerative medicine.
\end{abstract}

Keywords: rat bone marrow, mesenchymal stem cell, osteogenic, chondrogenic, adipogenic differentiation
Volume 3 Issue 3 - 2017

\author{
Sangeetha P,' Maiti S K, ${ }^{2}$ Divya Mohan,' \\ Shivaraju S,' Raguvaran R,' Malik Abu Rafee,' \\ Bindhuja BV,' Naveen Kumar,' Raguvanshi \\ PDS' \\ 'Division of Surgery, Indian Veterinary Research Institute, India \\ ${ }^{2}$ Division of Medicine, Indian Veterinary Research Institute, India \\ Correspondence: Swapan Kumar Maiti, Principal Scientist, \\ Surgery Division, Indian Veterinary Research Institute, \\ Izatnagar-243 122, Uttar Pradesh, India Tel +94|2049|69, Fax \\ $+9|58| 2303284$, \\ Emailswapanivri@gmail.com, maiti_62@rediffmail.com
}

Received: August 08, 2017 | Published: October 10, 2017
Abbreviations: rBM-MSC, rat bone marrow derived mesenchymal stem cells; CFU-F, colony forming unit-fibroblast; DPBS, dulbecco's phosphate buffer saline; DMEM, dulbecco's modified eagles medium; EDTA, ethylene diamine tetra acetic acid

\section{Introduction}

In the past decade, the field of stem cells and cell-based therapies has undergone a remarkable evolution. The potential of stem cell to differentiate into various types of cells has revolutionized their use in clinics for the treatment of a variety of clinical conditions. Mesenchymal stem cells can be cultured and grown for many generations under appropriate conditions in the laboratory and still retain a stable morphology and chromosome complement. ${ }^{1}$ Bone marrow obtained mesenchymal stem cells are the most studied stem cell type that is capable of differentiating into variety of cell lineages. Differences in differentiation ability to osteogenic chondrogenic and adipogenic lineages of MSCs harvested from Murine species of various age groups and the number of passage of these cultured cells has been reported. Osteogenic and chondrogenic potential reduced with each and every age group and adipogenic differentiation ability reduced only in cells obtained from oldest donors. ${ }^{2}$ The technique of bone marrow collection and stem cell culture vary for different species. ${ }^{3}$ Culturing of rodent bone marrow derived stem cell is a little bit difficult when compared to its human counterpart. ${ }^{4}$ Here we describe a simple and easy technique of stem cells isolation and differentiation from adult Wistar rats.

\section{Materials and methods}

\section{Bone marrow collection}

Two male Wistar rats of 14 weeks age were used for bone marrow collection. Prior permission was obtained from Institutional Animal Ethics Committee (IAEC) for conducting animal trials. Shortly after euthanizing the animal, back side extending from lumbar region to toes were shaved and aseptically prepared for dissection (Figure 1A). Skin incision was given on the lateral aspect of the thigh and both tibia and femur were exteriorized after removing the muscular and tendinous attachment (Figure 1B). Femur and tibia of both the legs were placed separately in a $50 \mathrm{ml}$ centrifuge tube containing DPBS (Dulbecco's Phosphate Buffered Saline) and antibiotics. It was then placed under laminar hood in petridish containing DMEM (Dulbecco's Modified Eagles Media) with antibiotics. With a scissor the metaphyseal region of both bones were cut. After inserting a needle into the medullary cavity the bone marrow was flushed out using DMEM into a $15 \mathrm{ml}$ centrifuge tube. Flushed out bone marrow was centrifuged for 5 minutes at $1000 \mathrm{rpm}$ in order to concentrate the cells. The supernatant was decanted and $5 \mathrm{ml}$ of complete media was added to the tube and mixed well by pipetting. Cell suspension was layered over equal quantity of histopaque and again subjected for centrifugation at $2500 \mathrm{rpm}$ for half an hour. After this density gradient centrifugation mononuclear cells were collected from the interface and washed with $\mathrm{Ca}^{++}$and $\mathrm{Mg}^{++}$free DPBS (Figure 1C). Obtained cell pellet was again washed and resuspended in complete DMEM (Figure 1D). Cell counting was done after addition of trypan blue dye in an automatic cell counter and was then seeded in T flask with a cell concentration of $1 \times 10^{6} \mathrm{cells} / \mathrm{ml}$. After 72 hours of seeding, nonadherent cells were removed by decanting the media. Every 3 or 4days media was replaced until the flask attained confluency.

\section{Culture specifications}

DMEM containing $15 \%$ fetal bovine serum (FBS) and $1 \%$ antibiotic mixture of $100 \mathrm{units} / \mathrm{ml}$ of penicillin and $100 \mu \mathrm{g} / \mathrm{ml}$ of streptomycin 
was used as growth media for the adherent cells. The culture flasks were maintained in a humid condition at $37^{\circ} \mathrm{C}$ with $5 \% \mathrm{CO}_{2}$. When the cells reached $80-90 \%$ confluency, Trypsin-EDTA solution $(0.25 \%$
Trypsin and 1mM EDTA) was added to passage the flasks. Further differentiation studies were performed with third passage cells.
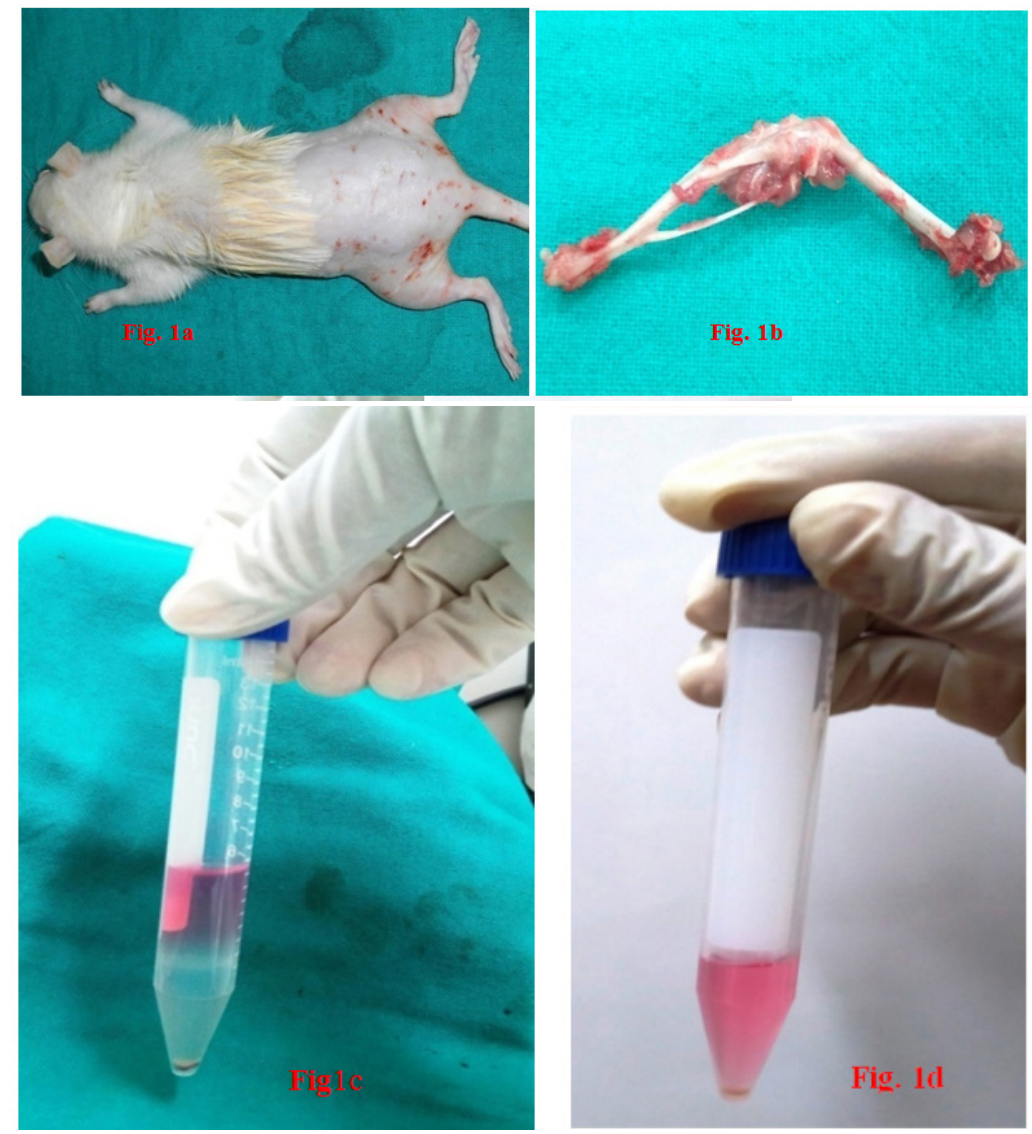

Figure I Collection and isolation of bone marrow from rat (IA: Preparation of surgical site. IB: Collection of femur and tibia. IC: Density gradient separation of buffy coat. ID: Centrifuged cell pellet).

\section{Colony forming assay}

A $100 \mathrm{~mm}$ tissue culture dish was plated with cells at a concentration of 2 cells $/ \mathrm{mm}^{2}$. Culture dish was kept in a humidified chamber at $37^{\circ} \mathrm{C}$ with $5 \% \mathrm{CO}_{2}$ and maintained up to 14 days. At the end of assay, DPBS was used to wash the dish and fixing of cells was done with methanol for 5 minutes. Cells were then stained with Giemsa stain for 30minutes. Finally, plates were washed with DPBS, dried and colonies were counted. Faintly stained ones were ignored.

\section{Assessment of cell viability}

3-(4, 5-dimethylthiazol-2-yl)-2, 5-diphenyl tetrazolium bromide (MTT) was used to assess the number of proliferating cells. Mitochondrial dehydrogenases present in the live cells will reduce MTT. Third passage cells were plated in a 96 well ELISA plate at a cell concentration of $1 \times 10^{4}$ cells/well with the addition of complete culture media and kept in an $\mathrm{CO}_{2}$ incubator at $37^{\circ} \mathrm{C}$ with $5 \% \mathrm{CO}_{2}$ for $0,3,7$ and 14days. After incubation $20 \mu 1$ MTT $(5 \mathrm{mg} / \mathrm{ml})$ was added to each well and incubated again for $4 \mathrm{hr}$ in $\mathrm{CO}_{2}$ incubator. At the end of incubation media was removed gently without disturbing the monolayer and was dried for few minutes. $150 \mu \mathrm{l}$ of DMSO was added to each well and mixed without formation of air bubble to dissolve formazan crystals. Absorbance was recorded at 570nm in an ELISA reader. DMSO was used as blank for the experiment (Figure 3C-3F).

\section{Characterization of rMSCs}

\section{Osteogenic differentiation}

Counted third passages cells were seeded in 24 well culture plate with a cell concentration of $2 \times 10^{6} \mathrm{cells} / \mathrm{well}$. When the cells attained $70-80 \%$ confluence, Osteogenic differentiation media (Stempro) was supplemented into four wells where as negative controls were supplemented with complete media. At weekly interval media was changed and culture was maintained up to 21 days. By alizarin red staining, calcium deposition was evaluated to assess the differentiation potential of cells.

\section{Adipogenic differentiation}

To stimulate adipogenic differentiation cell plating was performed as the same way as that of osteogenic differentiation. After attaining $70-80 \%$ confluency four wells were supplemented with adipogenic differentiation media (Stempro) and control wells received complete media. Media was changed twice in a week and culture was maintained for one month. Positive adipogenic differentiation was confirmed by oil red O staining which demonstrated lipid droplets inside the cell.

\section{Chondrogenic differentiation}

Cells were seeded as same as that for osteogenic and adipogenic differentiation. After attaining 70-80\% confluency, chondrogenic 
differentiation medium (Stempro) was supplemented into 4 wells and the control wells received complete media. Culture was maintained for 18days with twice weekly addition of media. Chondrogenic differentiation was assessed by Alcian blue staining.

\section{Results and discussion}

Mesenchymal stem cells are an attractive tool for therapeutic application due to its self-renewal capability. They are main source for tissue repair, tissue engineering and vehicles of cell-based gene therapy. ${ }^{5}$ Stem cells have been isolated from different types of tissues. Mesenchymal stem cells can differentiate into several tissue-forming cells such as bone, cartilage, fat, kidney, liver, tendon, muscle, heart and even brain cells. ${ }^{6}$ Although MSCs from human bone marrow are easy to harvest, the isolation of rodent MSC is much more difficult. The self renewing capacity and ability to differentiate into various functional cell types makes the mesenchymal stem cells an attractive and efficient tool for the field of regenerative medicine. Their homing ability and non immunogenic nature enhance their therapeutic usage. Bone marrow derived mesenchymal stem cells were isolated by density gradient centrifugation and their plastic adherent property was used for expansion. After initial seeding, the flasks were observed every three days. In the beginning cell population were rounded in shape. After three days cells got attached and those non adherent cells were removed by decanting the media. Supplementation of media was done at three days interval. Gradual change in the morphology of cells could be observed (Figure 2A-2J). Cells slowly changed into spindle shape. By 18 to 21 days $\mathrm{P}_{0}$ flask became confluent and was passaged with $0.25 \%$ trypsin EDTA solution. Addition of trypsin detached the cell monolayer and cells became rounded again. Centrifuged cell pellet was seeded into $T_{75}$ flask. Third passaged cells were used for differentiation studies. After 18-21days of culture, the $\mathrm{P}_{0}$ flask $\left(\mathrm{T}_{25}\right)$ became confluent which was further expanded by trypsinization. Thereafter, cells attained confluency within 4 to 5 days. $\mathrm{P}_{3}$ cells were used for in vitro studies.
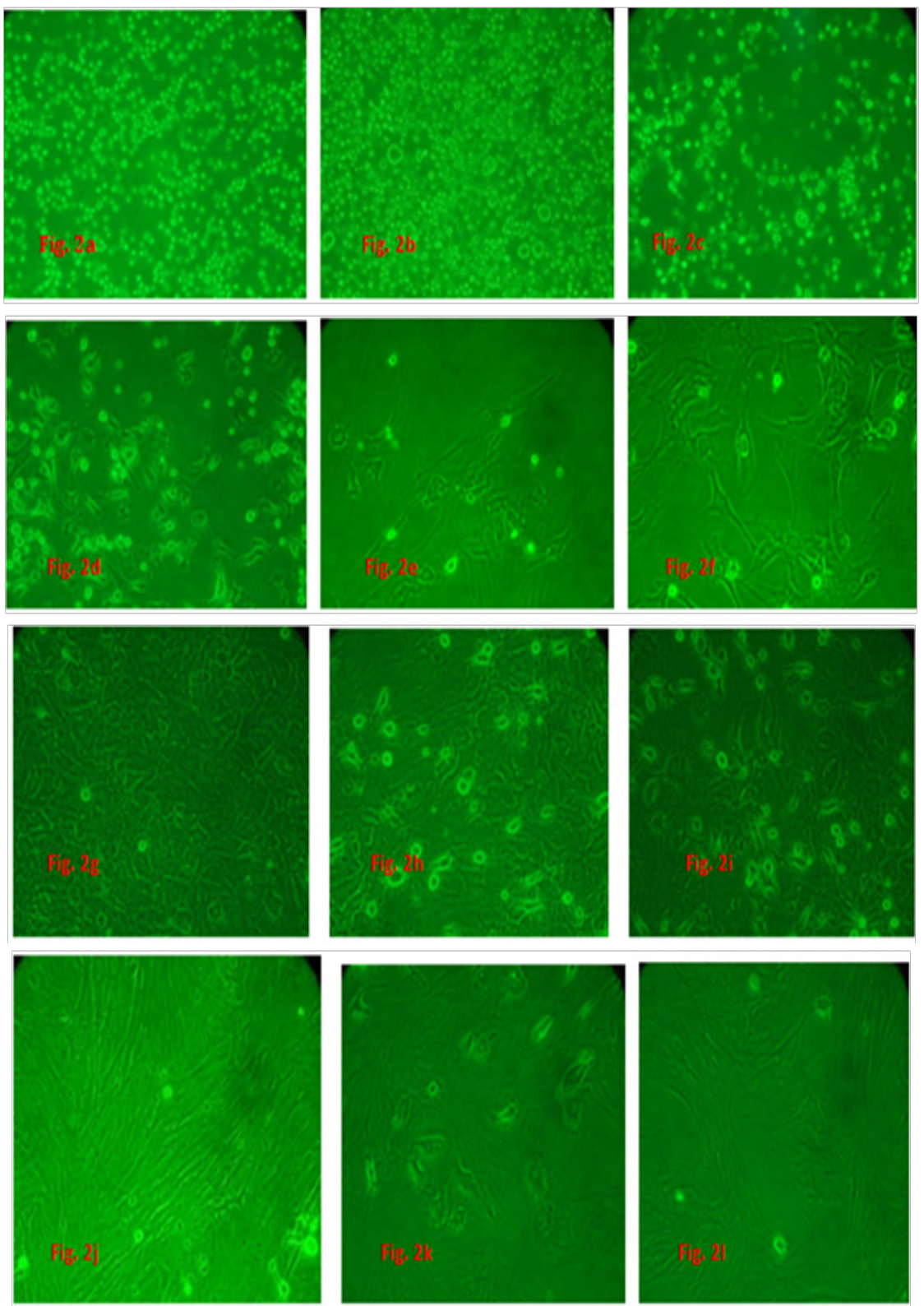

Figure 2 Culturing of rat bone marrow derived mesenchymal stem cells $\left(2 A: P_{0}\right.$ day 0. 2B: $P_{0}$ day 3. 2C: $P_{0}$ day 7. 2D: $P_{0}$ day 9. 2E: $P_{0}$ day I2. 2F: $P_{0}$ day I5. 2G: Confluent $P_{0}$ on day 18.2H: Confluent $P_{1}$. 2I: Confluent $P_{2}$. 2J, $K$, L: Confluent $P_{3}$ ). 


\section{CFU assay}

Colony forming unit-fibroblast (CFU-F) assay was used to assess the proliferation of cells and colonoigenic capacity of stem cells. ${ }^{8}$ Low density plating of cells resulted in the formation of colonies which was visible with Giemsa staining (Figure 3A,3B). Very little ECM present within the bone marrow allows mechanical disruption and dissociation of hematopoietic cells by simple pipetting and passage through syringe needles of decreasing bore. These single cell suspensions of stromal cells of bone marrow (BMSCs) when plated at low density could adhere rapidly to plastic surface. Non adherent hematopoietic cells suitable culture environment each of which is derived from a single precursor cell, the CFU-F. ${ }^{9}$ The mitogenic factors that stimulate the proliferation of CFU-F are not fully understood, but it is hypothesized that these include epidermal growth factor (EGF), insulin-like growth factor- 1 , transforming growth factor- $\beta$ plateletderived growth factor (PDGF) and basic fibroblast growth factor. ${ }^{10}$ Multi-colony-derived strains, where all colonies are combined by trypsinization, can undergo more than 25 passages in vitro (more than 50 cell doublings) under optimal culture conditions. This indicates the high self-replication capacity of BMSCs. Hence, millions of BMSCs can be produced from a limited quantity of aspirated bone marrow. Thus, BMSCs can be defined as cells which are adherent very rapidly with colonogenic property and able to proliferate extensively.

\section{MTT assay}

Cell viability of stem cells under standard condition was measured by MTT assay. ${ }^{11}$ MTT was reduced by the mitochondrial dehydrogenises present in the proliferating cells. Active enzymes in the live cells transform tetrazolium into intracellular formazan crystals. ${ }^{12}$ Cells were incubated with tetrazolium salts for 4 hours. Formazan salts became soluble after the end of incubation and absorbance was recorded at $570 \mathrm{~nm}$ in an ELISA reader. The absorbance will be proportional to the number of live cells in the wells that has reduced the MTT. Mitochondrial dehydrogenase was reduced by MTT and there was gradual increase in the absorbance at $570 \mathrm{~nm}$ on day 3,7 and 14 as compared to day 0 (Figure $4 \&$ Table 1 ). There was significant increase in the OD value of D14 in comparison to D7, D3 and D0. There was significant increase in OD value of D7 when compared to D0. OD value of D3 was non-significantly higher when compared to D0.

Table I Cell viability assay: Mean \pm SD of OD at $570 \mathrm{~nm}$

\begin{tabular}{cc}
\hline Days & Mean \pm SD \\
\hline D0 & $0.83 \pm 0.07^{\mathrm{a}}$ \\
D3 & $1.01 \pm 0.04^{\text {abd }}$ \\
D7 & $1.18 \pm 0.11^{\mathrm{b}}$ \\
DI4 & $1.64 \pm 0.13^{\mathrm{c}}$
\end{tabular}

Note: Statistically different values are with different alphabets.

\section{Differentiation studies}

Rat mesenchymal stem cells supplemented with osteogenic induction media could differentiate into osteogenic lineage after 21 days of culturing. Presence of extracellular mineralization was detectable on alizarin red staining. Positive osteogenic lineage showed intense red staining with alizarin red (Figure 3G) whereas, control culture showed light yellow background staining (Figure 3H). Adipogenic differentiation was detectable after 28days of culturing in adipogenic differentiation medium. By oil red $\mathrm{O}$ staining visible red colored lipid droplets was detected (Figure 3I). The control culture was negative for intracytoplasmic lipid droplets (Figure 3J). Chondrogenic differentiation of rat mesenchymal stem cells was achieved by supplementation with Stempro chondrogenic differentiation media. After 18days of culturing, differentiated chondrocytes with proteoglycans were positive for Alcian blue staining (Figure 3K) whereas, the control culture was negative (Figure 3L).

Rat mesenchymal stem cells underwent trilineage differentiation after culturing in specific induction media. Specific staining method revealed the ability of MSCs to differentiate. Trilineage capacity of rat MSCS was assessed by keeping cells in different induction medium compare to control media, isolated stromal cells were ability to differentiate into other mesenchymal cell lineages like osteoblast, adipocytes and chondrocytes. Osteogenic, adipogenic and chondrogenic differentiation potential was confirmed with special staining includes Alizarin red, Oil Red O, Alcian blue respectively. Calcium deposits in the matrix were demonstrated by alizarin red $\mathrm{S}$ staining. This histological staining is based on the capacity of alizarin red to specifically stain matrix containing calcium and its positive appearance is considered an expression of bone matrix deposition and hence confirmed osteogenic differentiation. Alcian blue staining of rat MSCs revealed the production of proteoglycans and hence confirmed the differentiation to chondrocytes. Oil Red $\mathrm{O}$ staining revealed intracellular lipid filled droplets that stained deep red confirming the adipogenic differentiation potential of rat MSCs. Expression of specific genes like Runx2, Ppar- $\gamma$ and Aggrecan for osteogenic, adipogenic and chondrogenic differentiation of rat MSCs through RTPCR was also reported. ${ }^{13}$ Stromal cells can take on many forms like cartilage, fat, bone or myelosupportive stroma. This character bone marrow derived stromal cells, both in vivo and in vitro, has possibly provided the first glimpse of the character which is now extensively called as plasticity. Clonal strains of bone marrow adipocytes can undergo osteogenic differentiation to form genuine bone in an in vivo assay. ${ }^{14,15}$ Previously, the capability of bone marrow reticular cells to change to adipocytes in vivo has been observed. ${ }^{16,17}$ Different studies have reported that fully differentiated chondrocytes could dedifferentiate and convert to osteogenic phenotype in culture, ${ }^{18,19}$ and similar phenomena can be identified in vivo. ${ }^{20}$ All these research findings suggest the non-irreversible nature of the differentiation of various cell populations otherwise seen as an end point of variety of lineages. Protocol for culture of mesenchymal stem cells derived from bone marrow of rabbit and other animal species was reported. ${ }^{21,22}$ Stem cell seeded biomaterials are now used routinely for different surgical affections. ${ }^{23,24}$ The primary implications of these research findings remained unnoticed until recently. Commitment and differentiation are not usually thought of as reversible, but rather as multistep, unidirectional and terminal processes. 

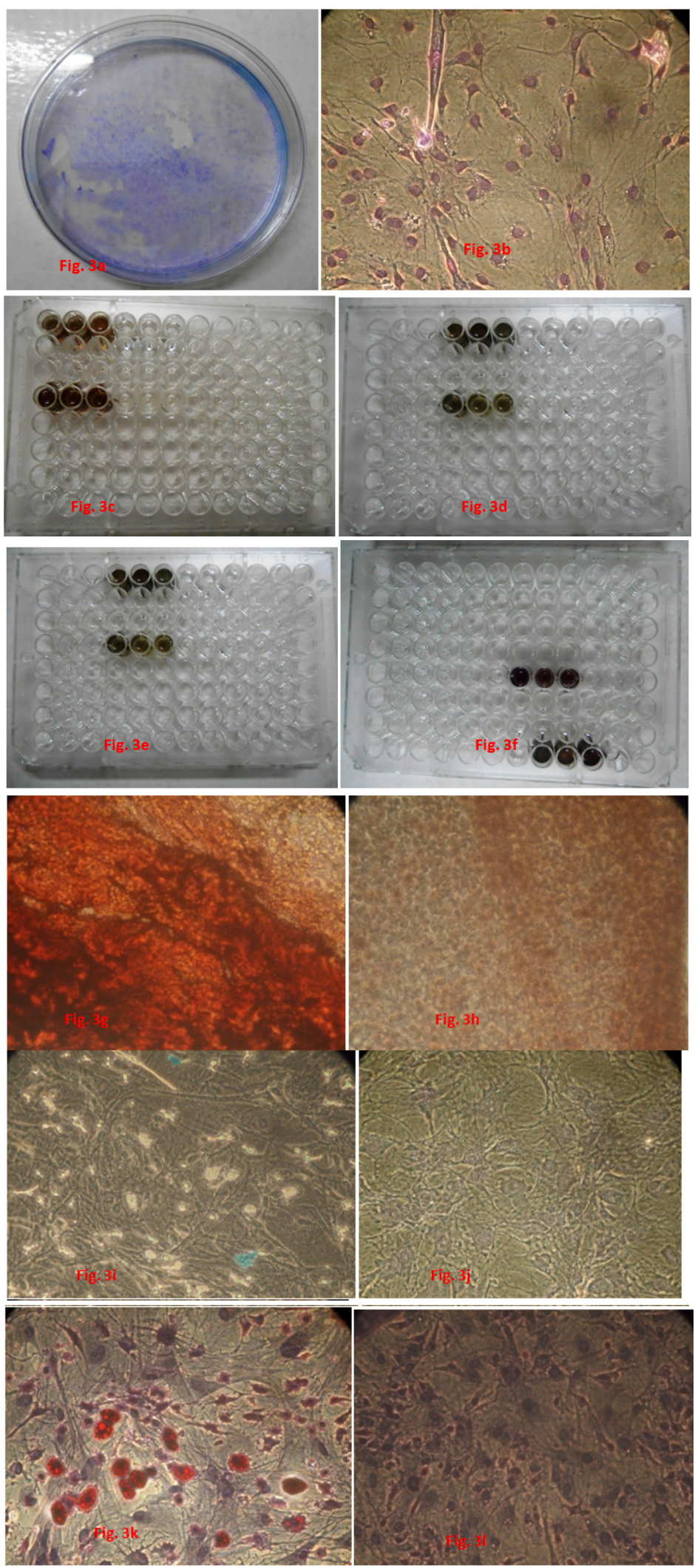

Figure 3 Differentiation of rat bone marrow derived mesenchymal stem cells ( $3 \mathrm{~A}$ and 3B: Colony forming assay. 3C, 3D, 3E and 3F: Cell viability assay on day 0, 3, 7 and I4 resepectively. 3G: Osteogenic differentiation positive with Alizarin red staining. 3H: Osteogenic differentiation negative in control with Alizarin red staining. 3l: Chondrogenic differentiation positive with Alcian blue staining. 3J: Chondrogenic differentiation negative in control with Alcian blue staining. 3K: Adipogenic differentiation positive with Oil red $\mathrm{O}$ staining. 3L:Adipogenic differentiation negative in control with Oil red $\mathrm{O}$ staining). 


\section{CELL VIAB ILITY ASSAY}

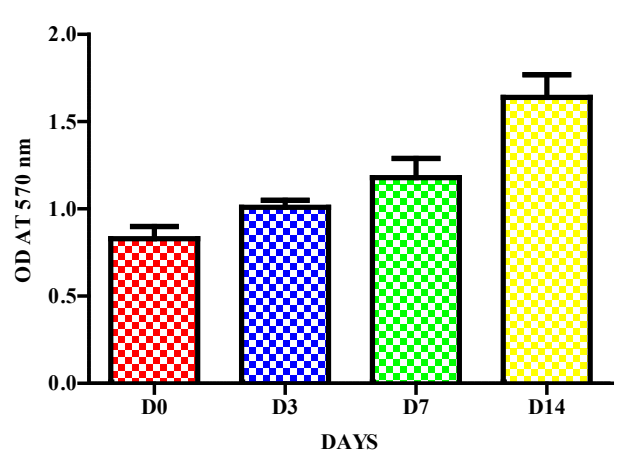

Figure 4 Cell Viability assay (Mean \pm SD of OD at $570 \mathrm{~nm}$ ).

\section{Acknowledgements}

The authors wish to thank to the Director, Indian Veterinary Research Institute, Izatnagar, India for providing necessary facilities for this study.

\section{Conflict of interest}

No conflicts of interests among the authors with respect to the authorship or the publication of this article.

\section{References}

1. Sandhaanam SD, Pathalam G, Dorairaj S, et al. Mesenchymal stem cells (MSC): Identification, proliferation and differentiation- A review article. Peer J PerPrints. 2013;1:e148v1.

2. Kretlow JD, Jin Y, Liu W, et al. Donor age and cell passage affect differentiation potential of murine bone marrow derived stem cells. $B M C$ cell biology. 2008;9:60.

3. Sekiya I, Larson BL, Smith JR, et al. Expansion of human adult stem cells from bone marrow stroma: conditions that maximize the yields of early progenitors and evaluate their quality. Stem Cells. 2002;20(6):530 541.

4. Eslaminejad MB, Nadri S, Hosseini RH. Expression of Thy 1.2 surface antigen increases significantly during the murine mesenchymal stem cells cultivation period. Development Growth and Differentiation. 2007;49(4):351-364.

5. Huang S, Xu L, Sun Y, et al. An improved protocol for culture of mesenchymal stem cells from mouse bone marrow. Journal of Orthopaedic Translation. 2015;3(1):26-33.

6. Alhadlaq A, Mao JJ. Mesenchymal stem cells: Isolation and therapeutics. Stem cells and Development. 2004;13(4):436-448.

7. Yoshimura H, Muneta T, Nimura A, et al. Comparison of rat mesenchymal stem cells derived from bone marrow, synovium, periosteum, adipose tissue and muscle. Cell and Tissue Research. 2007;327(3):449_ 462.

8. Pochampally R. Colony forming unit assay for MSCs. Methods in Molecular Biology. 2008;449:83-91.

9. Bianco P, Riminucci M, Gronthos S, et al. Bone marrow stem cells: Nature, Biology and Potential Applications. Stem cells. 2001;19(3):180192.
10. Gronthos S, Simmons PJ. The growth factor requirements of STRO-1positive human bone marrow stromal precursors under serum-deprived conditions in vitro. Blood. 1995;85(4):929-940.

11. Lu Y, Yuan Y, Wang X, et al. The growth inhibitory effects of mesenchymal stem cells on tumor cells in vitro and in vivo. Cancer biology and Therapy. 2008;7(2):245-251.

12. Lucarelli E, Beccheroni A, Donate D, et al. Platelet derived growth factors enhance proliferation of human stromal stem cells. Biomaterials. 2003;24(18):3095-3100.

13. Tamilmahan P, Maiti SK, Palakkara S, et al. Culture, characterization and differentiation potential of rat bone marrow derived mesenchymal stem cells. Journal of Stem Cell Research and Therapeutics. 2016;1(5):00034.

14. Bennett JH, Joyner CJ, Triffitt JT, et al. Adipocytic cells cultured from marrow have osteogenic potential. Journal of Cell Science. 1991;99(pt1):131-139.

15. Beresford JN, Bennett JH, Devlin C, et al. Evidence for an inverse relationship between the differentiation of adipocytic and osteogenic cells in rat marrow stromal cell cultures. Journal of Cell Science. 1992;102(pt2):341-351

16. Bianco P, Costantini M, Dearden LC, et al. Alkaline phosphatase positive precursors of adipocytes in the human bone marrow. British Journal of Haematology. 1988;68(4):401-403.

17. Weiss L. Haemopoiesis in mammalian bone marrow. Ciba Foundation Symposium. 1981. 84:5-21.

18. Gentili C, Bianco P, Neri M, et al. Cell proliferation, extracellular matrix mineralization, and ovotransferrin transient expression during in vitro differentiation of chick hypertrophic chondrocytes into osteoblast-like cells. The Journal of Cell Biology. 1993;122(3):703-712.

19. Galotto M, Campanile G, Robino G, et al. Hypertrophic chondrocytes undergo further differentiation to osteoblast-like cells and participate in the initial bone formation in developing chick embryo. Journal of Bone and Mineral Research. 1994;9(8):1239-1249.

20. Riminucci M, Bradbeer JN, Corsi A, et al. Vis-a-vis cells and the priming of bone formation. Journal of Bone and Mineral Research. 1998;13(12):1852-1861.

21. Ninu AR, Maiti SK, Shiva Kumar MU, et al. Isolation, proliferation, characterization and in vivo osteogenic potential of bone marrow derived mesenchymal stem cells (rBMSC) in rabbit model. Indian Journal of Experimental Biology. 2017;55(2):79-87.

22. Maiti SK, Shiva Kumar MU, Srivastava L, et al. Isolation, proliferation and morphological characteristics of bone marrow derived mesenchymal stem cells (BM-MSC) from different animal species. Trends in Biomaterials \& Artificial Organs. 2012;27(1):29-35.

23. Ninu AR, Maiti SK, Sangeetha $\mathrm{P}$, et al. In vitro osteoinduction potential of a novel silica coated hydroxyapatite bioscaffold seeded with rabbit mesenchymal stem cell. J Stem Cell Res Ther. 2016;2(1):0009.

24. Maiti. SK, Ninu AR, Sangeetha P, et al. Mesenchymal stem cells-seeded bioceramic construct for bone regeneration in large critical-size bone defect in rabbit. Journal of Stem Cells \& Regenerative Medicine. 2016;12(2):87-99. 